\title{
Academic exchange in the COVID-19 era
}

\author{
Yong-Ha Kim
}

Editor-in-Chief, Archives of Plastic Surgery

Department of Plastic and Reconstructive Surgery, Yeungnam University College of Medicine, Daegu, Korea

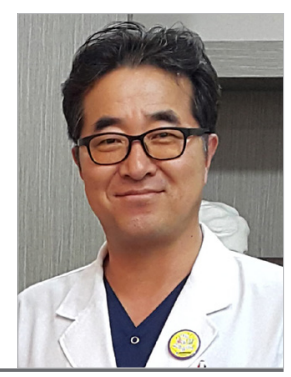

The entire world is in a state of emergency, as in wartime, because of coronavirus disease 2019 (COVID-19). The year 2020 will be known as one of the darkest periods in history, not only for industry, sports, and cultural events, but also for academic exchange. Most gatherings and exchange events, including academic conferences and seminars, have been postponed or canceled. Due to the lack of a vaccine or remedy for the disease, the only effective responses to the pandemic are wearing face masks, washing hands, and social distancing [1-3]. Therefore, most social interactions have been put on hold. Although the process of recovery has begun to a limited extent, it will take a considerable amount of time for face-to-face social activities to return to what they were like in the past. Academic exchanges, including academic conferences and workshops, are also in a state of general paralysis. Most likely, we will not see large exhibits, academic seminars, and receptions with thousands of attendees for the time being. As physical contact has become limited due to COVID-19, many workshops and resources are being provided online. Conferences are being replaced by online meetings, webinars, and online journal presentations. In fact, the more active role played by online delivery has positive ramifications for scholars who would like to achieve a deeper understanding of a certain discipline, because online seminars cost less and do not have space limitations, so they can disseminate information to a larger group of people.

The true purpose of large academic conferences is exchange. Academia advances through presentations, debates, arguments, and exchanges among scholars at conferences. During an academic conference, attendees have the opportunity to listen to lectures given by renowned experts, as well as presentations given by their peers, and to engage in debates with their colleagues. Scholars are exposed to and share a comprehensive range of ideas, from the most cutting-edge knowledge, traditional techniques, and new proposals to controversies or even dangerous ideas, theories, and results. Knowledge and techniques are not the only things that scholars share; attendees have a chance to network, thereby connecting personal and academic advancement. Thus, face-to-face contact with people in the same room was an important part of academic culture. When returning from a conference-by train or by plane-scholars would have the chance to savor the stimulation and inspiration from the conference, to reflect on memorable experiences and their enjoyment thereof, and to design the next leap forward in their research [4,5]. Scholars are now concerned that they may miss the heartwarming feelings of exchange and empathy they received from academic conferences as the COVID-19 pandemic persists.

Modern physics bloomed in the early 20th century in Germany, centered around quantum mechanics. A photograph of the Solvay conference in 1927 is one of the most famous pictures in any science book, as 17 of the 29 attendees eventually became Nobel laureates (Fig. 1). Through presentations, questions, answers, and arguments lasting 6 days, quantum mechanics become more theoretically sophisticated and formed the central axis of modern physics [6]. However, Hitler came to power in 1933. As the dictator implemented oppressive policies against Jews and used scholarly achievements for political purposes, the culture of exchange and advancement was destroyed. Many great scholars suffered from hardships on a personal level, and 


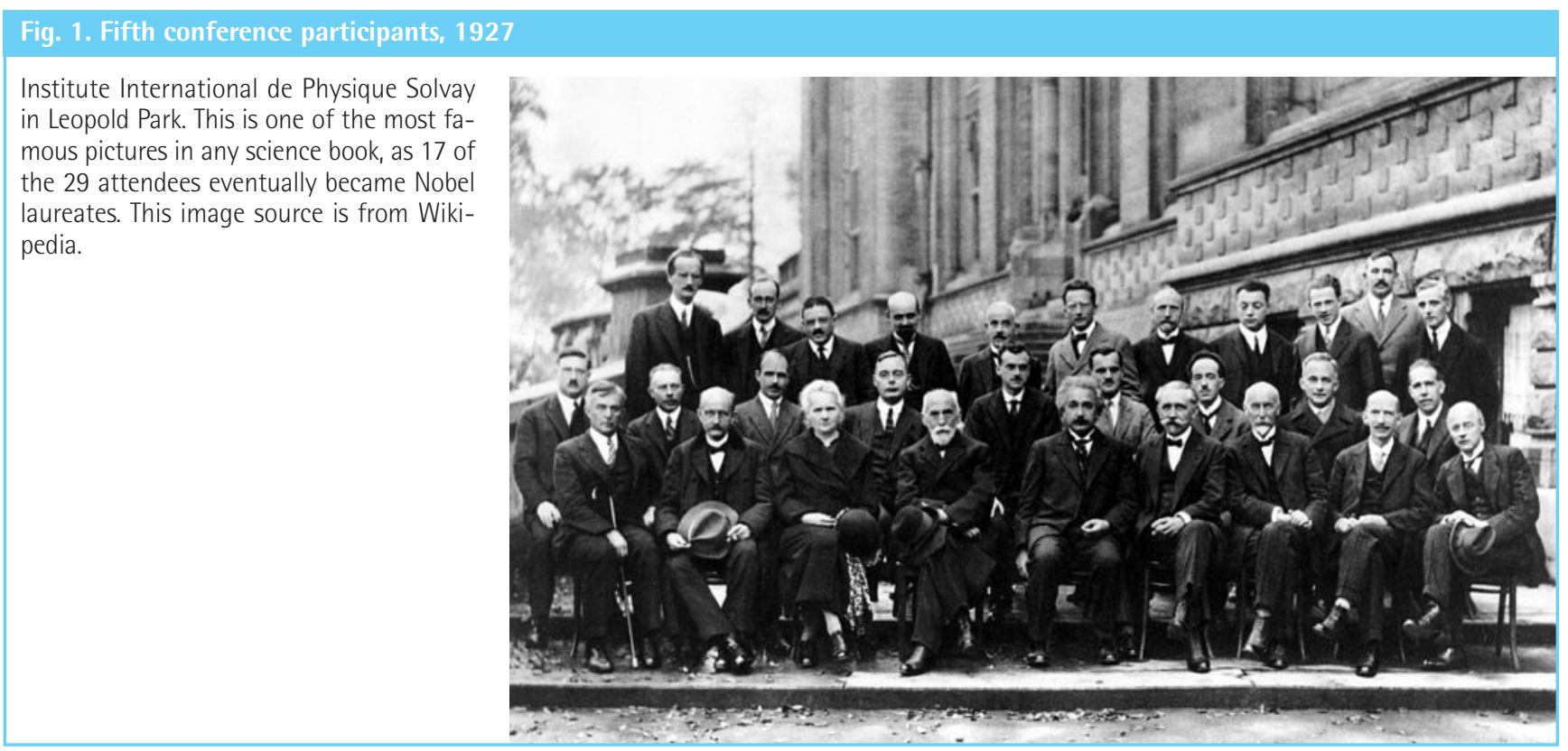

scattered all around the globe with their families. Some had to shift their intellectual focus, withdraw from their scientific careers, or face an unfortunate demise. Without Hitler, if the scholars who attended the Solvay conference had continued to conduct research and regularly meet at many seminars, I imagine that human civilization may have developed to a level that we could never imagine.

I worry that as the COVID-19 pandemic continues, it will have a lasting deleterious impact on advances in academia. I am concerned that, similarly to the advent of Hitler, the pandemic may destroy the culture of exchange and advancement among academics. I am rattled by the possibility that the prevailing sentiment might become to see others a fatal threat from which one has to keep one's distance, rather than as comrades with whom one can band together. I fear that the continuing severance of exchanges and prohibition of formal gatherings may strike a crippling blow to academic advancements, ushering the research community into a new dark age.

With the advent of COVID-19, many professionals are adjusting to the state called the "new normal," much different from their previous hectic and aggressive routines. Many have reduced their hours or are working from home to minimize physical human contact. Intradepartmental and interdepartmental meetings, external conferences, and social gatherings have mostly shut down. However, there are two sides to every coin. We now have more time to reflect personally. We have time to hone the theories we have been working on and to finalize papers that we have so far only been thinking about. Although this is a tough time, it could be a time of fermentation, which could

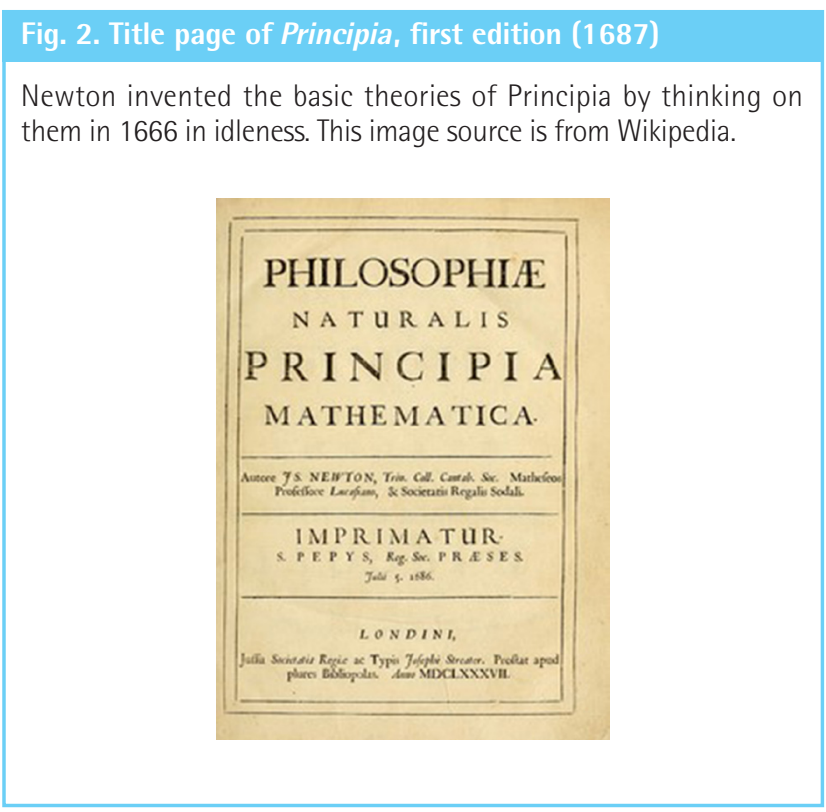

be a stepping stone for further leaps forward.

"In 1666, at the age of twenty-three, Newton was an undergraduate at Cambridge University when an outbreak of plaque forced him to spend a year in idleness in the isolated village of Woolsthorpe, where he had been born. He occupied himself by inventing the differential and integral calculus, making fundamental discoveries on the nature of light and laying the foundation for the theory of universal gravitation. The only other year like it in the history of physics was Einstein's "Miracle Year" of 1905 (Fig. 2) [7,8]." 
Although we are temporarily disconnected due to the pandemic, connections around the globe will never be fully severed, for finding ways to connect is the only way for us to live on [9]. We will need to maintain our connections and exchanges, while resolving our anxiety and managing risk. We will need to effectively implement remote delivery, such as online meetings, webinars, and online journal presentations. Therefore, I expect that the year 2020 will be marked by historical academic achievements despite the COVID-19 pandemic.

\section{NOTES}

\section{Conflict of interest}

YHK has served as an editor-in-chief of the Archives of Plastic Surgery, but have no role in the decision to publish this article. Except for that, no potential conflict of interest relevant to this article was reported.

\section{ORCID}

Yong-Ha Kim

https://orcid.org/0000-0002-1804-9086

\section{REFERENCES}

1. Hwang K. A safe distance between doctor and patient. Arch Plast Surg 2020;47:201-2.

2. Jang JG, Hur J, Choi EY, et al. Prognostic factors for severe coronavirus disease 2019 in Daegu, Korea. J Korean Med
Sci 2020;35:e209.

3. Jang WM, Jang DH, Lee JY. Social distancing and transmission-reducing practices during the 2019 coronavirus disease and 2015 Middle East respiratory syndrome coronavirus outbreaks in Korea. J Korean Med Sci 2020;35:e220.

4. Kim KS. Into a global future with our members to serve the people. Arch Plast Surg 2019;46:99-101.

5. Choi JW. PRS Korea 2017 \& 2018: how to organize an International Plastic Surgery Congress and how to make it more attractive. Arch Plast Surg 2018;45:295-7.

6. Wikipedia. Solvay Conference [Internet]. Wikipedia, Wikimedia Foundation; c2020 [cited 2020 Jul 6]. Available from: https://en.wikipedia.org/wiki/Solvay_Conference.

7. Sagan C. Cosmos. New York: Wings Books; 1980.

8. Wikipedia. Philosophiæ Naturalis Principia Mathematica [Internet]. Wikipedia, Wikimedia Foundation; c2020 [cited 2020 Jul 6]. Available from: https://en.wikipedia.org/wiki/ Philosophi\%C3\%A6_Naturalis_Principia_Mathematica.

9. Kim YS. Uncontact. Seoul: Publion; 2020.

Correspondence: Yong-Ha Kim

Department of Plastic and Reconstructive Surgery, Yeungnam University College of Medicine, 170 Hyeonchung-ro, Nam-gu, Daegu 42415, Korea

Tel: +82-53-620-3481, Fax: +82-53-626-0705, E-mail: kimyon@ynu.ac.kr

Received: June 24, 2020 • Revised: June 30, 2020 • Accepted: July 1, 2020

pISSN: 2234-6163 • elSSN: 2234-6171

https://doi.org/10.5999/aps.2020.01172 • Arch Plast Surg 2020;47:287-289 